Sādhanā Vol. 40, Part 2, April 2015, pp. 503-513. (C) Indian Academy of Sciences

\title{
Effect of fibre shape on transverse thermal conductivity of unidirectional composites
}

\author{
B RAGHAVA RAO ${ }^{1, *}$, V RAMACHANDRA RAJU ${ }^{2}$ and \\ K MOHANA RAO ${ }^{1}$
}

${ }^{1}$ Mechanical Engineering, VRS Engineering College, Vijayawada 520 007, India
${ }^{2}$ Mechanical Engineering, JNTU College of Engineering, Kakinada 533 003, India
e-mail: braghavarao@gmail.com

MS received 6 November 2013; revised 31 July 2014; accepted 6 November 2014

\begin{abstract}
The determination of thermal conductivities of a composite lamina is of paramount importance in the effective design and application of composite materials. The thermal conductivity of a lamina along the fibre direction can be easily estimated from the Rule of Mixtures but, the thermal conductivity in the transverse direction which depends on many factors need to be determined effectively. The transverse thermal conductivities of continuous fibre reinforced composite lamina are computed by numerical method using finite element analysis. Different fibre concentrations, fibre shapes and different fibre-matrix combinations are examined. A Regular array of square pattern of fibres is considered. The finite element model is validated with the available experimental results and theoretical models for a circular fibre and then extended to other shapes of fibres. Two-dimensional finite element model is adopted for the analysis, due to the restriction of heat flow only in transverse direction and the fibres are assumed to be continuous and perfectly bonded to the matrix. Analysis is carried out for a wide range of fibre-matrix combinations and up to the maximum fibre concentration in the composite. The analysis is extended for circular, square, elliptical and rhombus shaped fibres. From the results it is observed that there is a significant variation in the transverse thermal conductivity due to the shape of fibre, concentration ratios and fibre matrix combinations. This variation in thermal conductivity of a composite lamina results into a broader choice for the selection of composite materials in thermal applications.
\end{abstract}

Keywords. Transverse thermal conductivity; finite element analysis; square array; unidirectional composite.

*For correspondence 


\section{Introduction}

The prediction of macroscopic behaviour of composite materials is of fundamental and practical importance, as new composites are being developed continually for the automotive and aero space industries, electronic packaging, thermal insulation and many other applications. The performance of such materials, especially at elevated temperature environment depends quite heavily on the ability of the material to dissipate excessive heat. The overall heat conduction performance of these materials is thus a critical application parameter and worthy of research effort.

The problem of estimating overall thermal conductivities of composites is a sub group of more general field of determining effective transport properties of inhomogeneous systems from micro scale information. Due to the mathematical simplicity, this problem is closely related to other effective transport properties such as effective electric conductivity, dielectric constant, magnetic permeability whose relevant physical fields are governed by Laplace equation.

The spatial arrangement of fibres in the matrix is often modelled in two ways, periodic or random. Even though composite structures/systems with exact periodic fibre arrangement are not readily realizable, a periodic arrangement is assumed for developing mathematical models and to arrive at reasonable solutions. The rule of mixtures, $\mathbf{K}_{\mathbf{L}}=\mathbf{V}_{\mathbf{f}} \mathbf{K}_{\mathbf{f}}+\mathbf{V}_{\mathbf{m}} \mathbf{K}_{\mathbf{m}}$ works well in predicting the axial conductivity of a composite lamina as the resistance to the heat flow is constant. But, the resistance in the transverse direction of heat flow varies, and the transverse conductivity is to be evaluated more critically. Yet, this direction can be of great interest, since heat sources or an impinging gas or fluid on one side of laminate often creates a through temperature gradient. The work of this paper focuses entirely on this transverse thermal conductivity of the fibre reinforced composite lamina.

Rayleigh (1892) was the first to analyse the effective property of periodic arrays. He considered the effective electric conductivity of dilute dispersions with spheres arranged in a simple cubic array. His potential theory did not consider interactions of neighbouring fibres and hence limited to dilute volume fractions only. With the advent of composite media in engineering structures, interest in the effective conductivity was renewed. Beherens (1968) extended Rayleigh model for unidirectional continuous dispersions. Various analytical and numerical studies have been conducted to calculate the effective thermal conductivity using micro mechanics of composites by discretely modelling the fibre and matrix constituents. Most of these models were developed using a representative unit cell with regular arrays of fibre distributions, such as Square and Hexagonal array patterns of fibres and matrix material that fills the space among the fibres. Some considered irregular fibre distributions as well. The simplest micro mechanical model is Inverse rule of mixtures which does not consider the shape and geometrical arrangement of fibres, given by $1 / \mathrm{K}_{\mathrm{T}}=\mathrm{V}_{\mathrm{f}} / \mathrm{K}_{\mathrm{f}}+\mathrm{V}_{\mathrm{m}} / \mathrm{K}_{\mathrm{m}}$ underestimates the effective conductivity. Springer \& Tsai (1967) developed a unit cell model for square pattern fibre distribution and provided an analytical solution for the effective conductivity of a unidirectional lamina using shear analogy. The solution was obtained by dividing the unit cell into several sub domains and applying the rule of mixtures in serial and parallel combinations. Chamis (1983) with his simplified micromechanics model has obtained the same formula for square shaped fibre as Springer \& Tsai (1967). Zou et al (2002) used electrical analogy technique on square array unit cell model with third phase for the thermal barrier. They derived an analytical expression for effective thermal conductivity which reduces to Springer and Sai when the barrier effect is neglected. Hasselmen \& Johnson (1987) modified the original theories of Rayleigh and derived expressions for effective thermal conductivity of composites with a thermal barrier at the interface. But their work did not report a comparison with experimental data and their results are in better agreement only in dilute volume fractions. Perrins et al (1979) extended the formulation of Rayleigh to compute 
effective thermal conductivities of square and hexagonal arrays of infinitely long circular cylinders for the complete range of volume fractions and half complete range of conductivity ratio $\left(\mathrm{K}_{\mathrm{f}} / \mathrm{K}_{\mathrm{m}}\right)$ from 1 to $\infty$. The solution of Perrins matched very closely with the existing experimental results conducted on regular arrays of circular cylinders.

It is worthwhile to note that the various analytical solutions for predicting effective thermal conductivity of unidirectional fibre composites from the properties of fibre and matrix yield different results among themselves, especially for high volume fractions and high conductivity ratios. King et al (2008) conducted experiments and published the thermal conductivities using three types of carbon fillers with high conductive resins. Considering the above factors, it is highly difficult to determine the transverse conductivity experimentally and also obtaining analytical expressions as found in the literature. Only numerical models can accommodate all the above factors and provide better solutions which are more realistic. Developing numerical models with many parameters of influence becomes challenging and needs good theoretical and numerical skills.

In this study, we revisited and investigated the accuracy and validity of various micro mechanical models for the calculation of effective thermal conductivity. We proposed a numerical finite element model and validated its accuracy with the available analytical models and recent experimental data by King et al (2008). The purpose and novelty of this work is to find the effect of fibre shape on effective thermal conductivity with the assumptions that the fibre arrangement is square array and fibre and matrix are perfectly bonded and the composite is void free. Therefore, the parameters that effect effective thermal conductivity are thermal conductivity of constituents $\left(\mathrm{K}_{\mathrm{f}}, \mathrm{K}_{\mathrm{m}}\right)$, the fibre volume fraction and its shape. Researchers who worked in the area of estimating both mechanical and thermal properties of composite lamina/structures adopted circular fibres in their theoretical models and identified other parameters that influence the properties like content of the constituents, individual properties of the constituents, interfacial debond between the fibre and matrix and orthotropic nature of the fibre but none considered the effect of fibre shape. All the references cited in this paper also considered circular shaped fibres. It may be somewhat difficult to produce other shapes of fibres but the variations in the results certainly help the designer in critical areas of applications where property optimization plays a significant role not only in thermal, but also in combined loading conditions. This work has shown a different direction of getting a range of thermal conductivity with a change in shape of the fibre.

\section{Modelling of the problem}

In the present work, the transverse thermal conductivity of unidirectional fibre reinforced composites is studied by micro mechanical modelling using finite element method. The transverse conductivity of the composite lamina depends on many factors such as constituent properties, arrangement of fibres, content of fibre in the composite, contact between the fibre and matrix and the fibre shape. Since the purpose of this study is to evaluate the effect of fibre shape on the transverse conductivity, the other factors are assumed to be ideal/simple so that the desired effect is obtained. In this analysis, it is assumed that:

- Composite is macroscopically homogeneous.

- Locally both matrix and fibre are homogeneous and isotropic.

- Thermal contact resistance between the fibre and matrix is negligible. 


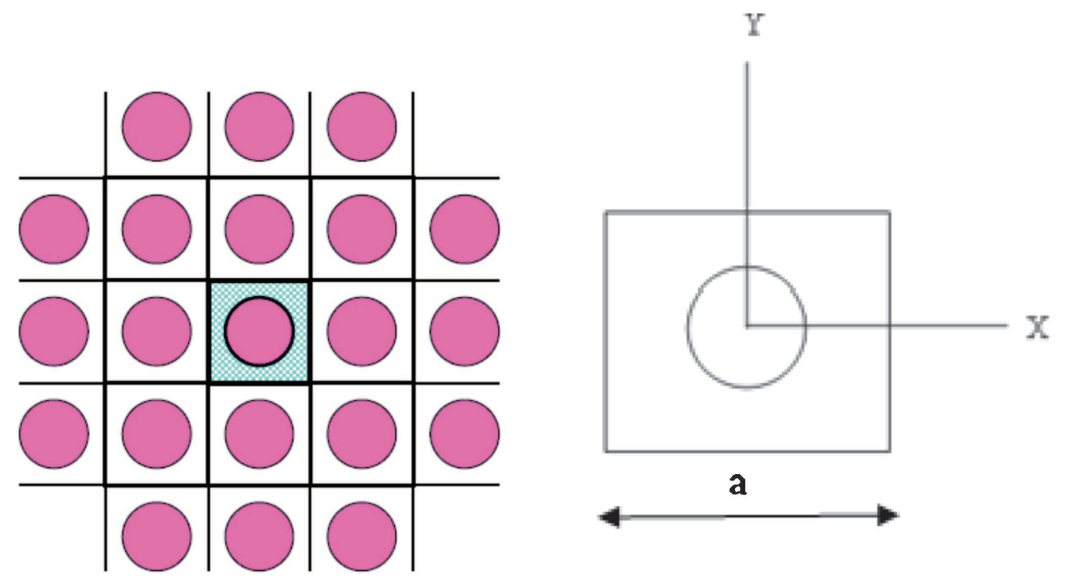

Figure 1. Square array and a representative volume element.

- The temperature distribution is independent of ' $\mathrm{Z}$ '- direction and hence the problem is 2-dimensional.

- The fibres are arranged in square periodical array.

The last assumption implies that the fibres are equal and uniform in size and shape and are symmetrical about $\mathrm{X}$ and $\mathrm{Y}$ axes (figure 1). This also results into only one transverse conductivity since $\mathrm{K}_{\mathrm{x}}=\mathrm{K}_{\mathrm{y}}$ for all the fibre shapes considered except the elliptical fibre.

A square unit cell $\Delta \mathrm{x}=\Delta \mathrm{y}=$ ' $\mathrm{a}$ ' is considered for the analysis and a temperature difference ' $\Delta \mathrm{T}$ ' is imposed across the cell in $\mathrm{y}$ - direction to calculate the steady state temperature distribution in the unit cell and hence the property of transverse conductivity. Han \& Cosner (1981) during their studies on thermal conductivities of composites for square periodic array fibre composites assumed one-dimensional (downward) heat flow which results into both the horizontal lines midway between fibres and the one passing through the fibre centers as isotherms and the corresponding vertical lines as adiabats. Hence a quarter unit cell (figure 2) can be used in place of a unit cell which reduces the problem complexity and computational time.

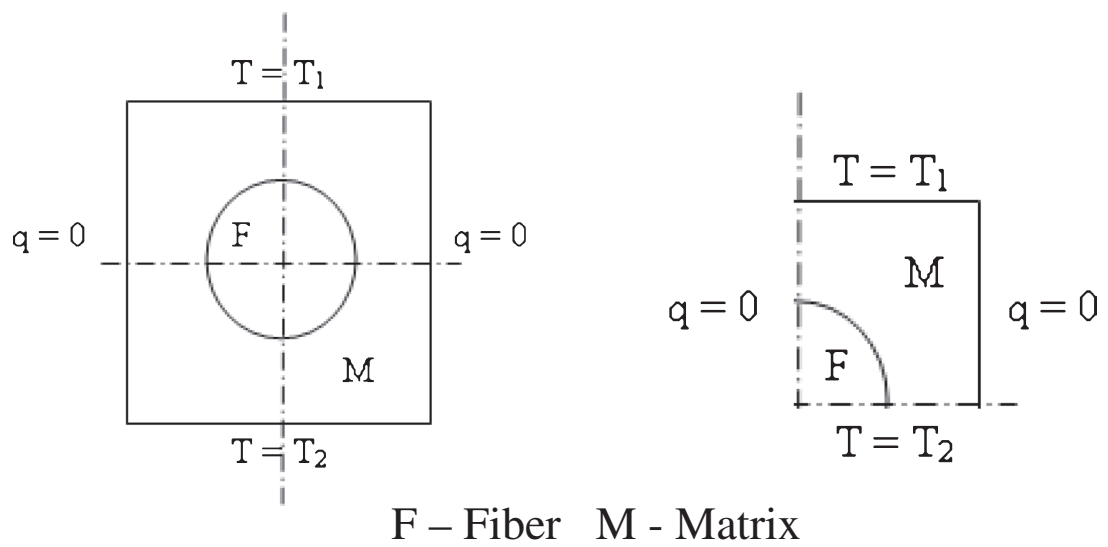

Figure 2. A unit cell and a quarter unit cell with boundary conditions. 
After a converged FE solution, the effective heat flux in Y- direction is calculated by integrating the heat flux distribution along the top of the unit cell. The transverse conductivity of the composite can then be obtained by

$$
\mathbf{K}_{\mathbf{T}}=\frac{\mathbf{q}_{\mathbf{y}}}{\left(\frac{\mathbf{d T}}{\mathbf{d}_{\mathbf{y}}}\right)} .
$$

In order to study the effect of various parameters on $\mathrm{K}_{\mathrm{T}}$, the results of composite transverse conductivity are normalized by the matrix conductivity such that $\mathrm{K}_{\mathrm{eff}}=K_{T} / K_{m}$ where $\mathrm{K}_{\mathrm{eff}}$ is dimensionless effective transverse thermal conductivity.

The steady state thermal problem is solved using finite element analysis software ANSYS. A 6-noded triangular element, plane 35 (figure 3(a)) is used to mesh the fibre and the matrix in the case of circular and elliptical fibres for a better fitment of curved geometries. An 8-noded quadrilateral element, plane 77 is used to mesh the fibre and matrix of square and rhombus shape. Temperature in the flow field, its gradient and the heat flow are the parameters that are sensitive to the finite element grid. However, since the thermal conductivity relates to both heat flow and the temperature gradient and therefore the variation of thermal conductivity with refinement of grid is studied and shown in the figure 3(b). The grid refinement study is done for all the fibre shapes and at all possible volume fractions of fibre and the conductivity ratios considered in the analysis.

The results of the analysis are compared with the results available in the literature and found to be in excellent agreement with the exact solution of Perrins et al who also conducted an
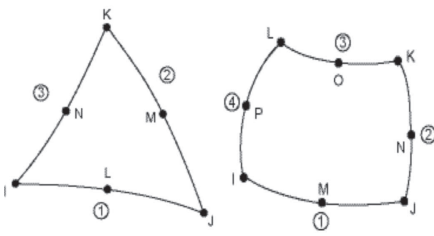

PLANE35

PLANE77
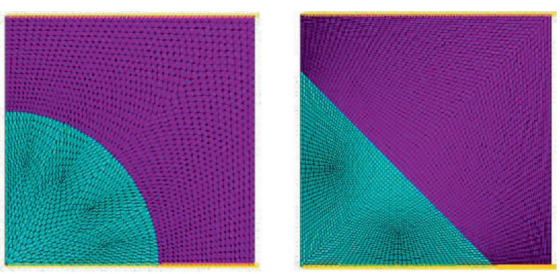

(a)

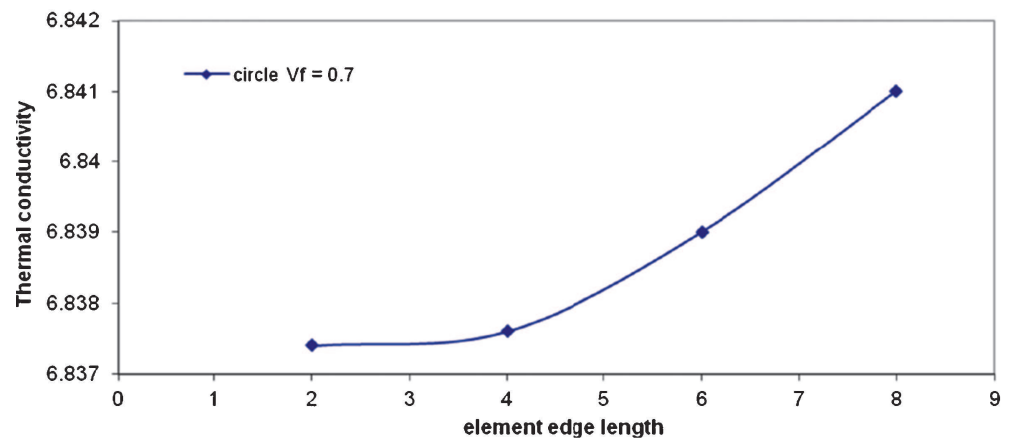

(b)

Figure 3. (a) Elements used in the analysis and refined mesh. (b) Convergence of thermal conductivity with refinement of grid. 

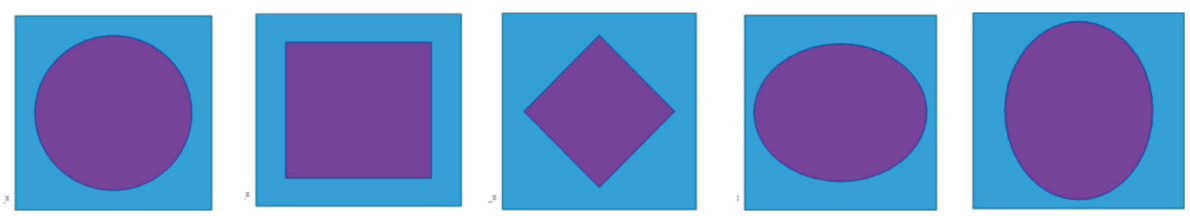

Figure 4. Different shapes of fibres used in the analysis.

experiment to support his theoretical solution. All the models used in the comparison are for circular fibre composites only.

The present model is extended for other shapes of fibres namely; elliptical, square and rhombus (figure 4). The analysis is carried out to study the effects of fibre geometry, fibre volume fraction and fibre matrix combination on the transverse thermal conductivity.

\section{Discussion of results}

To validate the model developed for the analysis, a comparison of the results for a circular fibre was made with the analytical and experimental results reported in the literature (figure 5). The graph shows a good agreement of the present finite element model with the reported results. The FE model deviated with experimental results particularly at higher volume fractions (beyond 0.35). In experimentation, fibres arrangement is not a regular periodic array as assumed but very much randomly distributed. At higher volume fractions of the fibre, this randomness causes some fibres to be in contact with others and hence a lower thermal resistance and a higher conductivity for the composite. Hence the model approximation of periodic array may be partly attributed for this deviation. The other factors which may also contribute to this are the non-homogeneity of

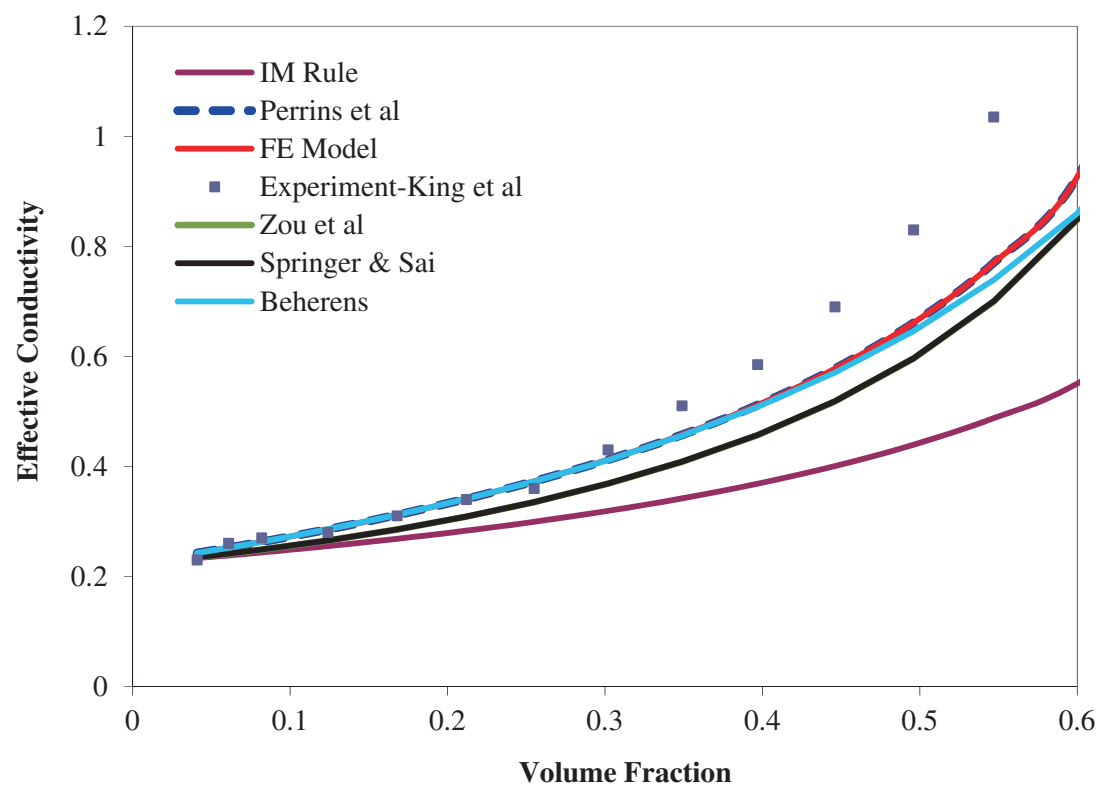

Figure 5. A comparison on transverse thermal conductivity of different models. 


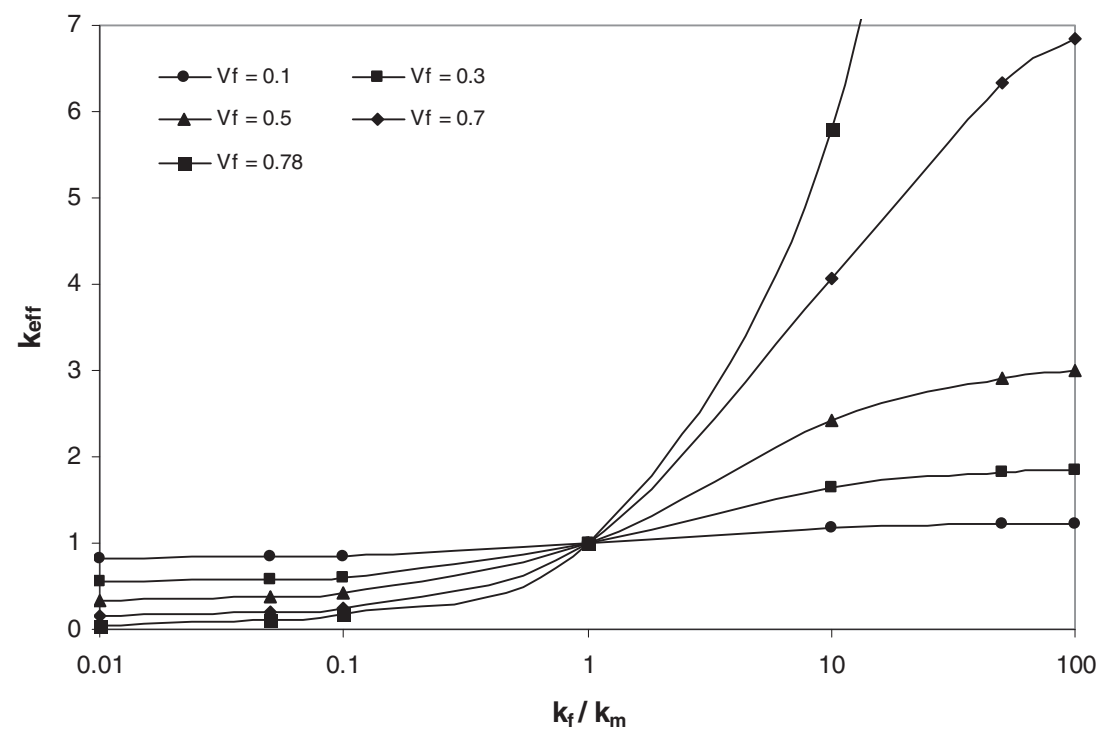

Figure 6. Variation of effective thermal conductivity in circular fibre composites.

the matrix and experimental inaccuracies. The effect of randomness on transverse conductivity is studied and highlighted by Sihn \& Roy (2011). They developed a random model and compared with the results of periodic models and shown a significant variation in the transverse thermal conductivity due to the randomness of fibre distribution especially at higher volume fractions of the fibre. Their random model shown a better agreement with the experimental data than the other periodic models and hence it can be concluded that the randomness is the major cause

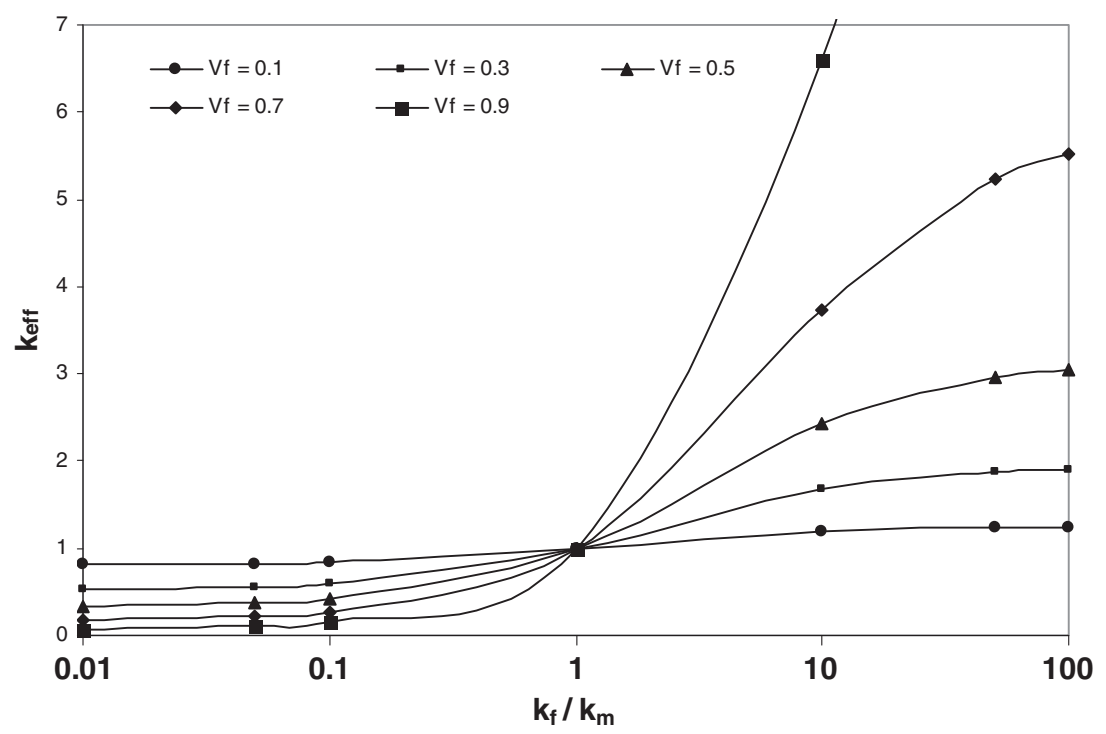

Figure 7. Variation of effective thermal conductivity in square fibre composites. 


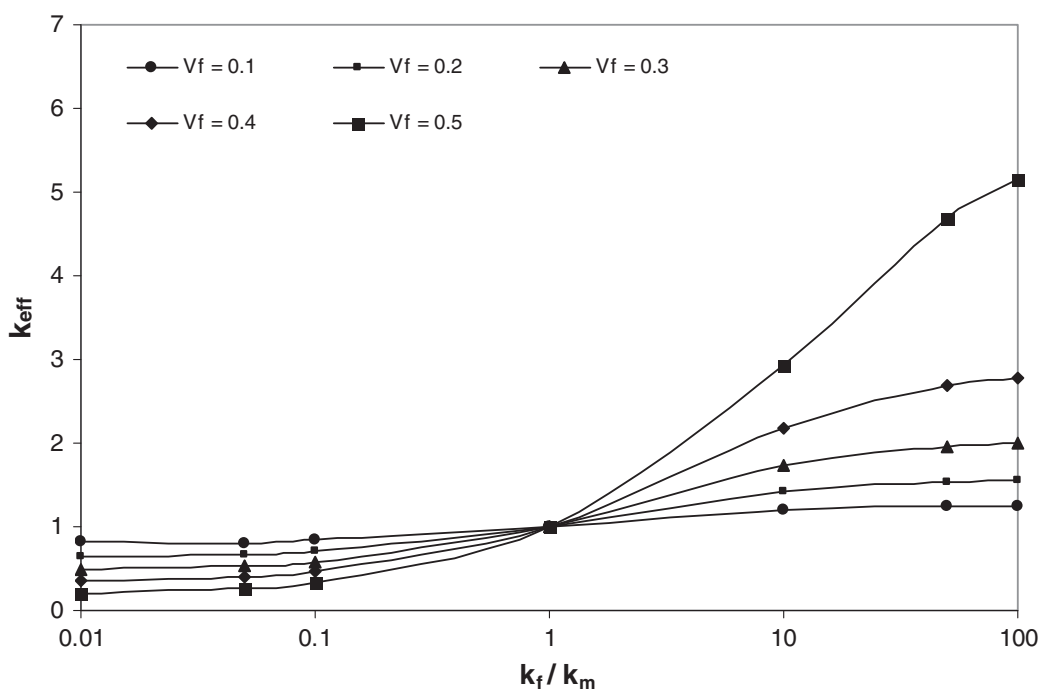

Figure 8. Variation of effective thermal conductivity in rhombus fibre composites.

for the deviation in the results of various models. This indicates that the present model can be extended to find the effect of shape of fibre by assuming a periodic array of fibre pattern.

The results for the effective conductivity for the four different shapes of fibres are plotted (figures 6-9). The range of fibre-matrix conductivity ratio considered is more realistic as reported in literature. The maximum volume fraction of fibre that can be accommodated in a unit cell strongly depends on the shape of the fibre which can be clearly observed in the above graphs. It can also be observed that there is a strong dependence of transverse conductivity not only on the conductivity ratio and volume fraction of its constituents, but also on the shape of fibre.

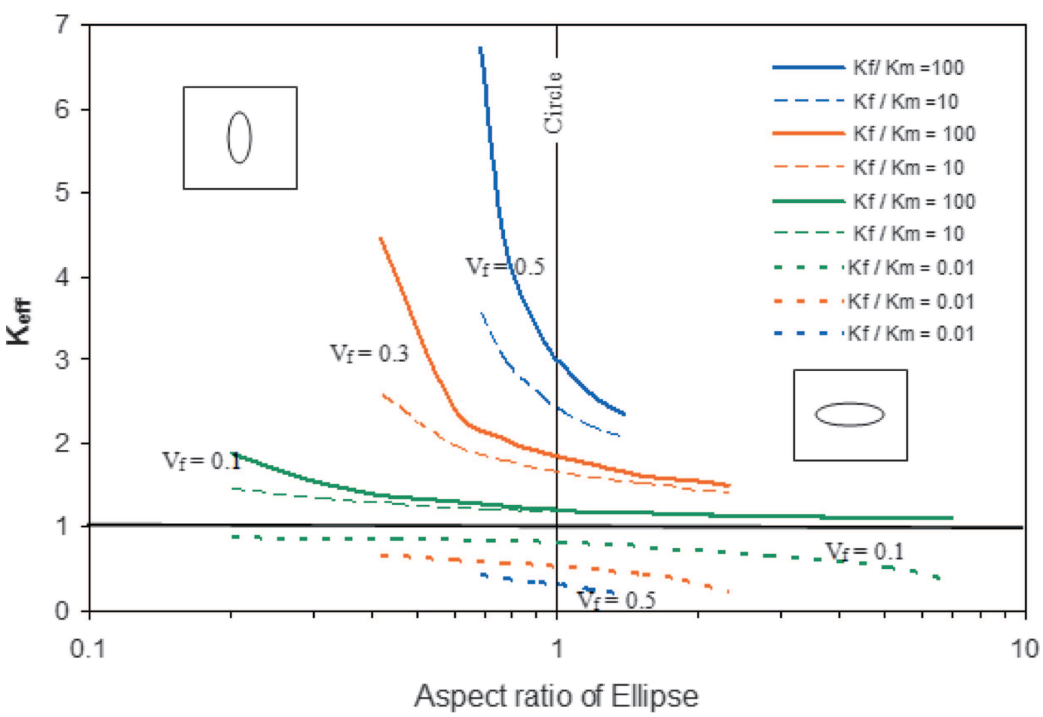

Figure 9. Variation of effective thermal conductivity in elliptical fibre composites. 
Ultimately, it is the combined resistance to the heat flow that determines the conductivity of any material. Since this combined resistance do change in a wide range with different shapes of fibres and hence are the large variations in the effective conductivity. In the case of an elliptic fibre (figure 9), there exists a possibility of two different arrangements by the fibre orientation as illustrated. Aspect ratio is defined as the ratio of major to minor axis and is considered as the influencing parameter in elliptical fibres. The results of the elliptical fibre clearly depict the
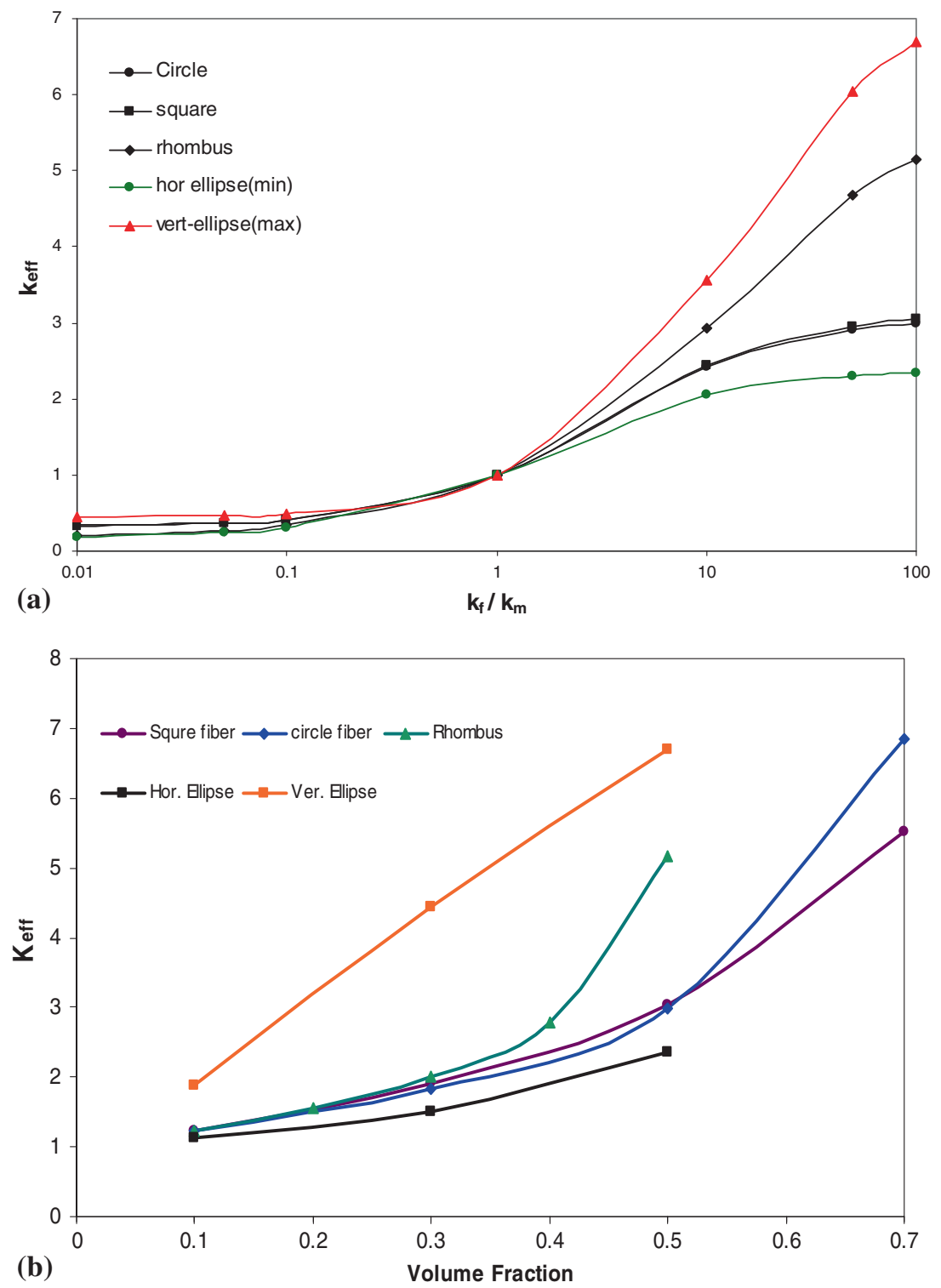

Figure 10. (a). Variation of effective thermal conductivity with constituents conductivity ratio in composites of different fibre shapes $\left(V_{f}=0.5\right)$. (b). Variation of effective thermal conductivity with fibre volume fraction in composites of different fibre shapes $\left(\mathrm{K}_{\mathrm{f}} / \mathrm{K}_{\mathrm{m}}=100\right)$. 
two possible different applications i.e., good and poor heat conduction in the two perpendicular directions which gives a greater flexibility in the thermal design of composites. For a better understanding and interpretation of the results, the variations of effective conductivity with conductivity ratio and fibre volume fraction for all the fibres considered are plotted (figure 10). It can be observed from these figures that the vertical elliptical and rhombus shaped fibre composites show higher conductivity. This is due to the higher heat flow path through the conducting fibre. With a similar reason, the lowest transverse conductivity is resulted in case of horizontal elliptical fibre composites. Hence an elliptical shaped fibre is a better choice in both conducting and insulating applications than the regularly used circular and square fibres. But it is also a fact that the maximum use of the elliptical fibres can not be achieved since this shape cannot accommodate higher fibre content in the composite. If the fibres in a composite represent a square pattern arrangement, then a maximum of $50 \%$ volume fraction is possible with elliptical and rhombus-shaped fibres and $78 \%$ for circular fibres and $100 \%$ for square fibres. Hence an optimization study is to be made before a final selection of the fibre shape which can give manufacturing simplicity and better structural and thermal results.

\section{Conclusions}

The transverse conductivity of a composite lamina which is important in many thermal related applications strongly depends on the materials considered for fibre and matrix and the amount of fibre in the composite. It can be concluded from the present work that the effective conductivity is strongly influenced by the geometry of the fibre also.

- The three shapes - circular, square and the rhombus exhibit transversely isotropic behaviour due to their geometrical symmetry. But the elliptical fibre shows a generally orthotropic behaviour and hence a greater design flexibility.

- The variation in effective conductivity at lower volume fractions is lower and at higher volume fractions is higher and this is true for all the shapes of fibres.

- The maximum volume fraction of fibre that a lamina can have depends strongly on the fibres pattern and also the shape of the fibre. For example, (a) with circular fibres, the square array can accommodate a maximum of $78 \%(\Pi / 4)$ and an hexagonal array can accommodate 90.9\% ( $\prod / 2 \sqrt{ } 3$ ). (b) By using square array pattern, circular fibres can achieve $78 \%$, square fibres $100 \%$ and rhombus fibres $50 \%$. In case of elliptical fibres this depends on ellipse aspect ratio and can reach a maximum which is equal to that of circular fibre.

- There is very little change in conductivity between circular and square shaped fibre composites up to $50 \%$ of fibre fraction.

- Among the fibre shapes considered in the present analysis, the vertical elliptical shape resulted highest conductivity and the horizontal elliptical shape the lowest. This is obvious from the fact that the thermal resistance for the heat flow is lowest and highest, respectively for the above two shapes. Hence the two shapes are preferable for good conduction and good insulation applications.

- Below $50 \%$ of fibre volume fraction, the vertical elliptical fibre composite shows a considerably higher conductivity compared to all other fibre shapes and hence is a choice in high conductivity situations.

- Because of the shape of fibre, the transverse conductivity can be raised by 1.5 times when the conductivity ratio is 10 and by 3.3 times when the ratio is 100 . These values are at generally used fibre fraction of 0.5 . 
Finally, it can be concluded that all the three parameters namely; fibre-matrix conductivity ratio, fibre volume fraction and the shape of the fibre need to be considered before a final selection of a composite material for thermal applications. A similar analysis may be made in the determination of other transport and elastic properties for a broader range of engineering applications.

\section{Notation}

$\mathrm{V}_{\mathrm{f}}$ Fibre volume fraction

$\mathrm{V}_{\mathrm{m}}$ Matrix volume fraction

$\mathrm{K}_{\mathrm{f}}$ Thermal conductivity of the fibre

$\mathrm{K}_{\mathrm{m}}$ Thermal conductivity of the matrix

$\mathrm{K}_{\mathrm{L}}$ Longitudinal (axial) thermal conductivity of Lamina

$\mathrm{K}_{\mathrm{T}}$ Transverse thermal conductivity of Lamina

qy Heat flux across the top surface of the quarter unit cell

'a' side of the unit cell

\section{References}

Beherens E 1968 Thermal conductivities of composite materials. J. Composite Materials 2(1): 2-17

Chamis C C 1983 Simplified composite micromechanics equation for Hygral, Thermal and mechanical properties. NASA TM 83320

Han L S and Cosner A A 1981 Effective thermal conductivities of fibrous composites. J. Heat Transfer 103: $387-392$

Hasselmen D P H and Johnson L F 1987 Effective thermal conductivity of composites with interfacial thermal barrier resistance. J. Composite Materials 21: 508-515

King J A, Hauser R A, Tomson A M, Wescoat I M and Keith J M 2008 Synergistic effects of carbon fillers in thermally conductive liquid crystal polymer based resins. J. Composite Materials 42(1): 91-107

Perrins W T, McKenzie D R and McPhedran R C 1979 Transport properties of regular arrays of cylinders. Proc. Royal Society of London Series A 369: 207-225

Rayleigh L 1892 On the influence of obstacles arranged in rectangular order upon the properties of a medium. Philosophical Magazine 34: 481-502

Sihn S and Roy A K 2011 Micromechanical analysis for transverse thermal conductivity of composites. $J$. Composite Materials doi: 10.1177/0021998310382311

Springer G S and Tsai S W 1967 Thermal conductivies of unidirectional materials. J. Composite Materials 1: $166-173$

Zou M, Yu B and Zhang D 2002 An analytical solution for transverse thermal conductivities of unidirectional fibre composites with thermal barrier. J. Phys. D: Appl. Phys. 35: 1867-1874 